\title{
Le motif du labyrinthe dans Le soleil des obscurs d'Abdelhak Serhane
}

\author{
Mounia Benalil \\ Université McGill
}

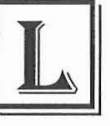

a littérature universelle foisonne d'exemples qui renvoient au mythe crétois du labyrinthe. Dans son livre Textes et labyrinthes, Philippe Forest étudie le fonctionnement de ce mythe dans des œuvres de James Joyce, Franz Kafka, Eduin Muir, Jorges Luis Borges, Michel Butor et Alain Robbe-Grillet. Selon Forest, bien que ces œuvres s'apparentent à la fable grecque du Minotaure, le motif du labyrinthe est plutôt exploité pour ses potentialités symboliques. Ainsi, la littérature moderne, occidentale surtout, réécrit le mythe archaïque autrement : elle réfléchit la condition du sujet moderne à partir d'une métaphore filée du labyrinthe : celle du monde (ou de l'existence) et celle du livre (ou de la bibliothèque). Il nous semble que le problème du héros moderne tel que Bakhtine l'approche dans son Esthétique de la création verbale à travers les trois dimensions de la spatialité ("Le tout spatial du héros »), de la temporalité (" Le tout temporel du héros ») et de la signification/signifiance ("Le tout signifiant du héros ») est envisageable dans la perspective de ce que Mircéa Eliade a, par ailleurs, nommé « l'épreuve du labyrinthe » (citée par Forest 10). Et dans l'histoire du réalisme, selon Bakhtine, la notion de l'épreuve, même si elle n'est pas nécessairement rattachée à l'idée du labyrinthe, est prédominante dans le type de roman dit d'apprentissage (ou Bildungsroman) où le parcours et le devenir du héros s'organisent à partir du principe structural de l'épreuve.

À lire Forest, on pourrait évoquer d'autres écrivains, non occidentaux, dont le recours au motif du labyrinthe s'inscrit dans le mouvement de l'interculturalité mondiale du discours romanesque. Le roman maghrébin contemporain convoque des lectures pertinentes dans la symbolique du labyrinthe. C'est le cas, entre autres exemples, de Tahar Ben Jelloun dont l'œuvre célèbre L'enfant de sable (et sa suite La nuit sacrée) se situe, dans sa 
démarche interculturelle, par rapport à l'œuvre de Borges, elle-même creuset dédaléen de cultures et de civilisations du monde. Chez d'autres auteurs, tels que Abdelhak Serhane, dont nous étudierons ici Le soleil des obscurs $^{1}$, le motif du labyrinthe organise la socialité du roman en faisant ressortir son intertextualité avec d'autres ouvres qui le dynamisent aussi.

Il nous semble que l'approche carnavalesque du Soleil des obscurs révèle une intersection entre la valeur mythique et la valeur symbolique du labyrinthe. Bien que l'image du labyrinthe se présente, à première vue, comme l'antithèse de l'image du carnaval, car si l'une évoque l'enfermement et l'errance, l'autre évoque l'éclatement et le projet subversif (donc une victoire sur l'errance), nous pouvons affirmer avec Forest que c'est au niveau du motif et de son usage littéraire qu'il est théoriquement possible de repérer certaines caractéristiques qui, dans le motif, sont à la base même du roman carnavalesque : il s'agit de l'hétérogénéite du motif, de son principe corporel et de sa chronotopicité (transposition de l'espace et du temps).

Le soleil des obscurs offre quelques espaces de lectures où la situation labyrinthique revendique l'action carnavalesque. Le roman raconte la vie des adolescents du milieu traditionnel marocain, notamment d'un jeune couple, Soltane (15 ans) et Mina (13 ans), coincé dans son épanouissement entre le poids de la théologie populaire et le diktat de la loi patriarcale. À travers l'histoire de ce couple, c'est le pays qui est mis à l'épreuve de sa gestation historique vers le renouveau identitaire et démocratique. Joël Des Rosiers a écrit dans un dossier spécial, "La médecine et la littérature », de la revue Spirale que certains « écrivains contemporains, attentifs aux pièges et aux pouvoirs du récit médical, cherchent à s'insérer dans le sillage d'une médicalisation de la culture et à renouer peut-être avec la tradition » (2000 : 12). Abdelhak Serhane, psychologue et écrivain, propose, à travers le réalisme mordant et dérangeant du Soleil des obscurs, un véritable diagnostic de la société marocaine de type traditionnel à son état pathologique. Le relais, au niveau romanesque, entre le discours psychanalytique (latent) et le discours socio-critique (manifeste) inscrit le texte de Serhane dans une littérarité où l'idéologique rencontre le pathétique.

À travers trois volets d'analyse, nous explorerons la valeur du schéma labyrinthique et de sa carnavalisation dans le roman de Serhane, et ce dans

1 Le soleil des obscurs sera désigné dans le texte par le sigle SO. La publication de cette étude est rendue possible grâce à une subvention de recherche postdoctorale du Fonds québécois de recherche sur la société et la culture (FQRSC). 
la perspective de la tension entre l'épreuve de la théologie et l'épreuve de la modernité dans le développement du sens identitaire. La carnavalisation des foyers labyrinthiques dans le roman constitue le pivot de l'idéologème de " médicalisation " de la culture traditionnelle marocaine.

\subsection{Labyrinthes et théologie populaire dans Le soleil des obscurs}

Le roman s'ouvre sur l'image de l'errance dans un labyrinthe physique et psychologique sans issue : un village est frappé de sécheresse et des jeunes adolescents s'ingénient à expliquer cette conjoncture en rapport avec leurs écarts de conduite en matière de pratique religieuse. Le sens de l'épreuve, du châtiment, de la fatalité, de la culpabilité et du péché nous place dans l'univers de la tragédie et présage le catastrophisme subséquent du roman. La situation labyrinthique est absurde et son interprétation en tant que "signe du ciel » (11) structure tout le roman. Cette interprétation permet de distinguer entre les univers opposés des adultes et des jeunes adolescents. L'architectonique de l'échange entre les deux parties est à son degré zéro et le dialogue y est presque absent. La structuration binaire des deux univers donne lieu à deux foyers de perceptions qui nous renseignent sur le savoir et la pratique théologique dans le Maroc traditionnel : celui des aînés, imbus de religiosité, et celui des adolescents qui grandissent loin de toute religiosité. Au fil du roman, « le sentiment religieux ${ }^{2}$ » s'érige en un macro-labyrinthe qui imprègne d'autres micro-labyrinthes au sein desquels s'effectuent, paradoxalement, l'évolution, l'intégration et la socialisation du sujet, notamment ceux des institutions religieuses (la mosquée), sociales (la communauté) et politiques (l’État). La mise en abyme de chaque micro-labyrinthe dans le macro-labyrinthe renferme elle aussi une mise en abyme de l'action carnavalesque de sorte qu'il est possible de supposer que le dispositif carnavalesque à l'œuvre dans le schéma labyrinthique agit comme force subversive de celui-ci. Dans le roman, "le sentiment religieux » conditionne la vie des jeunes perdus dans un néant chronotopique où s'abîment leur humanité et leur héroïsme : "Nous perdions petit à petit la foi et abondonnions toutes les valeurs et

2 Par référence au livre de Jean Déjeux, Le sentiment religieux dans la littérature maghrébine de langue française. Paris : l'Harmattan, 1986. L'auteur englobe sous le vocable de "sentiment religieux ", " la religion (musulmane, pour la grande majorité des écrivains) avec la foi spécifique, les institutions, les rites, les valeurs morales, de même que les pratiques non canoniques populaires " (19). 
toutes les qualités qui faisaient de nous des êtres humains, des personnes fières et dignes. [...] Perdus et inutiles [...], [nous sommes des] perpétuels angoissés dont l'existence et le devenir sont entre les mains du hasard, de l'imprévu, de l'imprévisible " (SO 13-16). Si " le sentiment religieux " retient sa valeur anthropologique, il serait trop simpliste et même redondant de poser que son questionnement par l'auteur vise à rendre la religion directement responsable des maux dont souffre la société marocaine traditionnelle. Le discours socio-critique s'appuie sur la religion en tant que "donnée " culturelle opératoire dans l'entreprise romanesque de Serhane comme dans celle de la majorité des écrivains maghrébins qui sont dans, ce que Déjeux appelle, "l'islamité ", c'est-à-dire une " manière d'être au monde et de se sentir musulman, avec les racines et les valeurs culturelles ainsi que le degré de participation au groupe, sans que le "musulman » soit tenu de rendre compte alors d'une foi éventuelle en un Dieu transcendant. " (1986: 27). La textualisation du "sentiment religieux » est un repère nécessaire dans l'organisation du sens du texte autour de la double thématique identitaire et religieuse.

Le traitement romanesque du " sentiment religieux " à travers le discours des institutions pré-nommées ainsi que du statut du sujet dans l'institution " montre que le religieux [chez Serhane] connaît un déploiement double : l'officiel, qui mesure son épaisseur et contrecarre sa dispersion et le populaire, qui le tourne, le retourne, le décode et l'encode selon ses différentes subjectivités "(Chikhi, 1996 : 217). Ce premier partage entre l'officiel et le populaire en matière de religion signe la mouvance carnavalesque du roman, dans la mesure où la culture populaire, telle que Bakhtine la présente dans son Rabelais, se greffe sur la culture savante pour effectuer le renversement subversif des choses. Le soleil des obscurs se situe à l'intersection de ces deux dernières formes de l'islam syncrétique. L'islam savant, strictement fondé sur les préceptes du Coran et de la charîa (loi islamique), est très diffus dans le roman contrairement à l'islam populaire qui est largement folklorisé. On parlera donc d'une représentation romanesque de la religiosité et non pas de la religion dans le Maroc traditionnel; la religiosité englobe toutes les formes d'expressions et de pratiques non canoniques de l'islam officiel.

\section{I.I L'INSTITUTION RELIGIEUSE : LA MOSQUÉE}

Lieu de culte musulman et symbole d'une autre institution qui lui fait pendant, l'école coranique, la mosquée est le lieu " privilégié de la contrebande du rêve et de l'illusion " (SO 48). Des " aveugles " (34) y

\section{2 * LittéRéalité}


chantent le Coran et l'imam y assume une tâche ambivalente : il est l'allié des autorités politiques et le représentant de la parole d'Allah (Dieu) auprès des croyants. C'est du point de vue des adolescents que nous percevons le lieu saint de l'islam. Pour échapper au rétrécissement quasi-kafkaesque de l'espace sacré, les jeunes s'évadent par l'imagination, Soltane pense à son futur mariage et son père, Si Larbi, à Aouïcha, son amour perdu (34-48). La mosquée devient le lieu d'épanchement des pensées subversives ou joyeuses. Elle se dépouille de sa sacralité : Si Larbi, y évoque les déboires de Aouïcha et de leurs aventures au moment où les jeunes scrutent l'entrée mystérieuse d'un colibri qui "s'acharnait sur le crâne chauve de l'imam [...] [et ayant] percé un trou [...] réussi à extraire la fibre de mensonge qui s'y trouvait » (39-40).

La prière collective prend une tournure carnavalesque lorsque le sermon " monologique » (39) de l'imam, hissé à une " dignité » subversive grâce au manège de l'oiseau, devient critique de l'ordre; les femmes quittent leur espace réservé dans la mosquée et s'aventurent parmi les hommes pour exécuter " une danse frénétique sous le regard comblé des jeunes garçons. [...] Les corps gagnés par la transe s'immobilisèrent [...] [et] [1] a multitude grouillante se dispersa dans la confusion » (44-45) à l'arrivée des "flics déguisés en croyants [qui] tentèrent d'interrompre le spectacle et de mettre fin à cette situation [...] pouv[ant] dégénérer en révolte " (43). La parole sacrée est politisée, dégagée de ses accessoires métaphoriques et elle se fait critique du pouvoir oppresseur et du conditionnement des consciences. L'imam, dans son " délire " (43) de lucidité, dénonce sa complicité avec les autorités et informe ses auditeurs, dans un grotesque eschatologique, du châtiment qui, dans l'au-delà, sera réservé aux personnes qui abusent du pouvoir. Or pour les jeunes qui se laissent "prendre au jeu de l'espoir possible " (49) de la parole sacrée et de sa " douce mélodie " (48-49), il reste à vérifier si les nouvelles revendications de l'imam ne sont pas une autre manière de détourner le vide plutôt que d'y remédier.

\section{I.2 L'INSTITUTION SOCIALE : LA COMMUNAUTÉ}

Dans le roman, l'islam populaire se manifeste dans les valeurs du groupe et du sentiment communautaire. Du point de vue des jeunes, « otages consentants des adultes, de l'autorité et des traditions » (89), aller contre "l'intérêt du groupe » et de " la cohésion communautaire » (76), présente un risque d'exclusion $(12 ; 63)$ et de marginalité totale. Ainsi bouclés dans le vivier de "la morale millénaire » (76) des ancêtres, les jeunes font l'offrande absolue 
d'eux mêmes (104) : étant des " roseaux pensants » et révoltés sans véritable pouvoir d'agir, ils acquiescent à jouer le jeu des adultes et demeurent prisonniers d'une rêverie velléitaire de changer les choses.

Plusieurs situations nous renseignent sur l'islam populaire dans le Maroc traditionnel. Cet islam recouvre une diversité de pratiques allant de la superstition au maraboutisme, pratiques traduisant la " cumulation dialoguée de formes variées de religiosité qui constitue la spécificité de l'islam maghrébin " (Ferchiou, 1996 : 6). Le syncrétisme foncier de l'islam populaire signe sa non-canonicité et son hétérodoxie. Le culte des saints est un phénomène marquant de l'islam populaire. Dans le roman, ce culte se traduit par le rituel de l'intervention du $f q^{2} h^{3}$ guérisseur dans le vécu des gens, que ceci soit pour remédier à un mal encouru ou pour contrer l'éventualité d'un événement indésirable.

Le mariage constitue l'événement le plus important où le recours au fqih est inévitable. Serhane décrit le mariage comme une affaire de groupe; la communauté y intervient de plein droit sur tous les plans et l'individu y est rarement un pouvoir de contestation. C'est l'étape " nécessaire à [l'] intégration définitive [de chacun] dans le groupe des hommes; un rite de passage de l'enfance à l'état adulte " (SO 84). Tout au long du roman, $\mathrm{Si}$ Larbi, «maudit le groupe qui l'avait contraint [à épouser sa cousine plutôt que Aouïcha] [...], et à croire le contraire de ses convictions intimes " (136). Sa mère avait consulté des marabouts pour empêcher son mariage avec Aouïcha, convaincue que l'attachement de son fils à cette femme ne pouvait être que l'ouvre de la magie (38). Pour la communauté, Aouïcha est une "femme fatale " (37); son pouvoir de séduction est dangereux pour l'ordre de la communauté (77) qui l'a sévèrement bannie. À sa disparition mystérieuse dans un puits, elle épousa, selon la croyance populaire, le démon (76) et devint la légendaire Aïcha Kandisha, "reine des Eaux " aux pouvoirs illimités que les "femmes opprimées " (18) viennent implorer au pied d'un " olivier creux" (17). Sur un registre surnaturel, elle incarne la voix de l'oracle qui prédit à Soltane son destin funeste (19) et prend régulièrement " possession de son corps et de son esprit " (18) à chaque fois qu'il rentre en transe (131) de révolte contre son mariage arrangé (95) et qu'un fqih est convoqué pour l'exorciser (131-32). Lorsque Mina

3 Pluriel de foqaha. Le fqih est « un personnage ancien dans la société marocaine, dont la légitimité et le savoir sont religieux. Il est censé pouvoir réciter le Coran par cour et connaître les ahãdith [leçons du Prophète]. Les foqaha [...] ne sont pas des docteurs de l'islam, à l'instar de ceux formés à l'école Quarawiyyin qui possèdent le titre officiel de "álim ou de faqîh " (Saâdia Radi, 1996 : 192). 
rencontre Aouïcha au bain maure (SO 201) et lui raconte le drame de ses menstrues, jusqu'alors incomprises (213), Aouïcha lui organise des séances avec des fqihs et collabore avec eux pour guérir, à travers leur " écriture sacrée » (216), le « mal affreux » (215) des menstrues perçues, du point de vue de la jeune mariée, comme une œuvre satanique (215).

Tous ces exemples montrent que « l'islam en tant que référence contrôle un champ de croyances bien plus vaste que l'islam en tant que dogme " (Radi 199). La solidarité de la communauté repose sur le partage d'un " islam qui parle à l'imagination et au cœur, alourdi de supersititions et de folklore " (Delcambres, 1999: 73) plutôt qu'un islam orthodoxe ou austère. La description de l'islam populaire dans le Maroc traditionnel permet à Serhane d'explorer les archétypes refoulés de la société marocaine, à travers la coupure du religieux et du social. Si Serhane s'élève contre le baroquisme de la pratique religieuse, c'est parce que celle-ci s'avère responsable de l'obscurantisme dans lequel se complaît la majorité des masses populaires. À travers la question de la théologie populaire, Serhane transpose, comme on le verra plus loin, plusieurs tensions psycho-pathologiques qui sont la traduction des multiples mutations que vit la société marocaine. Lorsque le psychopathologique de l'écriture " accomplit et prend en charge ce que la société n'assume pas, toutes les coupures de principe et d'ordre nécessaires à la redynamisation de la communication et des relations sociales " (Chikhi, 1996 : 161), apparait, alors, l'infirmité du discours théologique populaire face à la problématique du corps dans le labyrinthe de l'interdit et du tabou qui lui sont imposés. Le corps se dévoile en tant que zone symptomatique par excellence de la misère morale et matérielle du peuple.

\section{I.3 L'institution POLITIQUe : L'ÉTAT}

La critique de l'État marocain ponctue tout le roman de Serhane. La contestation de la conjoncture socio-politique marocaine, dans le roman, pourraît être légitimée par la textualisation romanesque d'un verset coranique récité par l'imam subversif lors de la prière collective du vendredi : "Nous avons offert au ciel, à la terre, aux montagnes, le dépôt de la foi; ils ont refusé de s'en charger; ils ont tremblé de le recevoir. L'homme s'en chargea; et il est devenu injuste et insensé » (SO 39). La dénonciation des tares de la société marocaine et de l'abus de pouvoir qu'exercent ses dirigeants se vérifie par le verset coranique; "le dépôt de foi » transgressé renvoie, dans le contexte socio-politique du roman, à l'irresponsabilité et à la négligence des dirigeants quant aux devoirs qui leur incombent dans la Cité musulmane. 
L'institution politique et les différentes lois qui la gérent tiennent plus de l'héritage jâbilite (ou pré-islamique) que proprement islamique. Cela se voit dans la féodalité des rapports entre les gens du peuple et les représentants de l'autorité politique ainsi que dans l'échec du projet démocratique après l'indépendance du Maroc en 1956. Le roman est très représentatif des inégalités accrues entre les riches et les pauvres, entre les aisés du système, les " négriers ». (200) et leurs sujets, les gens du peuple, les « esclaves » (200) héritiers méconnus de la lutte anticoloniale. Dans le village, le représentant du pouvoir est le nouveau caïd qui «s'est accapar[é] toutes les terres fertiles et [...] les terres qui étaient à l'abandon [...] [et] [a] mobilis[é] une centaine de fellahs pour les travailler» (182-83). En s'octroyant le droit d'irrigation des terres acquises avec « la bénédiction de l’autorité centrale » (183), le caïd agit en colon, exploitant et déshéritant les paysans de leur legs collectif, la terre des ancêtres. Dans cette vaste " pyramide des tyranneaux » (Memmi, 1972 : 38) où chaque administrateur agit en "petit colon ", le flic, le postier, le gendarme, l'instituteur, le douanier, l'épicier, le gouverneur, etc. (SO 18182), le caïd n'est " qu'un échantillon de cette vaste politique de déshumanisation des masses menée par l'État» (189). Pour Soltane, « [f]ils d'une terre blessée, orpheline de ses propres racines » (188), le refus de céder son bout de terre au caïd est un " acte d'indépendance » (186) qu’il exprimera jusqu’à son départ pour la Grande Ville.

La politique de déshumanisation dont parle Serhane assez ouvertement et à laquelle il consacre son essai Les prolétaires de la haine (1995) est le reflet d'une transition mal vécue entre la décolonisation et la réforme démocratique du pays. Ici, Le soleil des obscurs fait écho au Soleils des indépendances (1970) d'Ahmadou Kourouma, œuvre charnière où il dénonce, avec la même rigueur de l'ironie corrective, les maux de la société africaine et la corruption de ses politiciens. Si dans l'esprit simpliste des paysans l'absence de la « justice du ciel » (SO 7), c'est-à-dire le manque de pluie, est la conséquence "logique » de l'injustice terrestre $(17,38)$, pour Serhane, elle est la métaphore du fatum du sous-développement et de la subordination dans lesquels l'État maintient le peuple.

La démocratisation est autopsiée par l'auteur en tant que pure dystopie. La parole démocratique pourrait ressembler à une parole du sacré qui n'aurait subi, au cours de l'Histoire, aucun ijtihad (effort intellectuel de recherche et d'interprétation), une parole abstraite et figée qui ne trouve aucun ancrage dans le réel. "La Démocratie », expliquent les adolescents, " avait retrouvé le langage des siècles aveugles [...] du destin des pierres quand l'histoire, aveugle et sans scrupule, donne aux mirages le visage de la vérité et de la réalité » (64-65). La démocratie marocaine (ou à la maro-

\section{6 * LittéRéalité}


caine) est mâtinée de féodalisme; ses facettes sont multiples, ayant pour dénominateur commun une certaine "illusion référentielle » (Riffaterre 1982) : le discours démocratique des dirigeants ne signifie pas en relation aux choses du réel car entre le réel exposé (par l'auteur) et la parole démocratique qui le dit (celle des dirigeants), il y a l'espace du blanc, du brouillage et de l'occultation mensongers (SO 193) d'un véritable programme démocratique. Sous le vocable à double versant de la démocratie, l'auteur inclut toutes les formes de délire et de désarroi social que traverse la société marocaine " prise en otage par sa propre légende et victime de ses contradictions séculaires » (85). Serhane qualifie cet état de choses de " temps de chien " (135), temps de la " nausée », de la " peste " et de la " chute" (dans le sens théologique et existentiel que Sartre et Camus donnent à ces notions respectivement dans La nausée, La peste et La chute) qui se poursuit en accéléré jusqu'au Deuil des chiens (1998), dernier roman de l'auteur où la représentation du réel surpasse le tragique du Soleil des obscurs.

\subsection{Le corps-labyrinthe/le corps dans le labyrinthe}

Discuter la question du corps dans Le soleil des obscurs revient à discuter les mythes et les pratiques de la sexualité au Maghreb. Serhane a consacré une analyse psychanalytique fouillée à cette question dans son essai L'amour circoncis (1995) et aussi bien Le soleil des obscurs que d'autres romans antérieurs de l'auteur, notamment Messaouda (1983), peuvent être lus comme des versions romancées de son étude sur la sexualité au Maroc. À travers une telle fictionnalisation se déploie la réalité des rapports entre hommes et femmes dans le contexte ostracisant de la tradition. L'auteur a choisi d'éclairer cette "zone d'ombre " ou zone du h'chouma (tabou) dans un langage décapant qui ne reproduit ni les clichés de l'érotisme des Mille et une Nuits ni les promesses exotiques des harems orientaux. Par contre, le roman garde et interroge ce que Chebel appelle « l'esprit de sérail ", c'està-dire un cadre traditionnel d'organisation des rapports " aussi bien pour la femme que pour l'homme, lequel doit constamment démontrer sa parfaite socialisation, sa performance dans tous les domaines et son souci d'amélioration " (1995: 201).

Dans le labyrinthe multiforme des institutions religieuses et sociales surtout, la réflexion sur le statut du corps achemine sa " rencontre polyphonique avec le mal " (Sjursen 1995). Le roman de Serhane poursuit cette rencontre cynique dans la perspective globale d'une action sur l'atonie culturelle dans laquelle sombre la société marocaine traditionnelle. Une telle poursuite finit toujours par rencontrer ce qui la barre : l'accomplissement 
amoureux, car dans le roman, et à différents degrés, aussi bien le corps masculin que le corps féminin est nié, carencé et frustré. Et l'être qui porte ce corps est, lui aussi, comme en jachère, inculte et évidé, en un mot, il est l'incarnation de l'ignorance et de toutes les insuffisances. Le discours romanesque aborde la sexualité masculine et féminine dans ses anomalies, en tant qu'expérience limite de l'étrangeté et de la souffrance. Chez Serhane, comme chez Ben Jelloun, «nommer le sexe et l'exhiber [sont des] manière[s] de profaner le code doxologique grâce auquel fonctionne la société patriarcale. [...] [Cette] pratique sacrilège, provocatrice et subversive [...] est une manière de désigner l'archaïsme social " (Gontard, 1993 : 153). Les rapports interpersonnels sont presque inexistants et aussi bien les adultes que les jeunes sont dépeints dans la réclusion solitaire des fantasmes sexuels.

La sexualité masculine est traversée par l'inhibition, la déviance et le souci de la virilité. C'est dans le fantasme que Si Larbi revit ses " ébats amoureux » (SO 32) avec Aouïcha tout en violentant le corps de sa femme, Lalla Batoul $(72-73 ; 121)$; c'est dans l'homosexualité que Sidi Bouali, père de Mina, trouve une satisfaction inédite à ses penchants fantasmatiques (55; 75); c'est dans l'intransigeance que le patron de Soltane trouve une compensation à son impuissance sexuelle (196) et c'est dans le voyeurisme érotomaniaque que le caïd du village asseoit son autorité (230). Du point de vue des jeunes, "les adultes trouv[ent] leur harmonie dans la contradiction " (75-76). Cette contradiction qu'ils appellent, par ailleurs, par " puritanisme hypocrite " (89) leur échappe du moment où ils sont seuls à faire leur apprentissage et à trouver des réponses encore oiseuses aux questions qui les taraudent en matière de sexualité : " nous manquions d'expérience et les adultes étaient trop hautains pour donner quelque crédit à notre délire. Chez nous, les garçons naissent adultes et leur existence est dépourvue de caresses et de tendresse. Nous vivions avec la rumeur, dans la rumeur, et nous apprenions ses caprices " (101). C'est dans la rue qu'ils font l'apprentissage des "rudiments " (84) de la sexualité. Leurs propos montrent l'angoisse du corps féminin, affublé de tous les surnoms dévalorisants qui renvoient au cauchemar d'un vagin-labyrinthe denté et avalant (79). L'ignorance projette les jeunes dans un univers imaginaire où tout se confond, l'amour avec le viol, les pulsions avec la violence.

Le roman se focalise sur Soltane qui, comme ses jeunes camarades, n'échappe pas au drame de l'ignorance. Astreint à sa condition de corps et paralysé par l'angoisse de son mariage, Soltane vacille entre une transposition incestueuse de ses désirs sur le corps de sa mère (100) et la 
canalisation perverse de ses pulsions à travers la masturbation (73) et la zoophilie $(59-61 ; 88)$. Dans le grotesque et la monstruosité tramés de ces pratiques aberrantes apparaît l'extrême misère sexuelle des jeunes dans le milieu traditionnel marocain. Il résulte de ce refoulement du désir et de cette solitude des corps une quête contraire à l'amour de la femme et une intériorisation de la virilité en critère absolu d'une masculinité monocorde, ne se définissant pas par rapport à son corollaire, la féminité, mais soustraite à la sensibilité et à la douleur de la femme. La peur et la haine du corps féminin demeurent insurmontables pour les jeunes (99) du roman tout comme le souci de la performance sexuelle pour Soltane lors de sa nuit de noces dont il avait le " programme en tête " sans en saisir la moindre "signification" (84).

Le traitement de la condition de la femme traditionnelle au Maroc et de sa sexualité dans le roman magnifie l'idée de l'anéantissement du sujet puisque le personnage féminin est le plus charcuté par le patriarcat. " La subversion du discours rituel ${ }^{4}$ ", populaire ou religieux, repose sur la représentation de la femme en tant que double "agent de rupture » et de "libération par l'imaginaire» (Nisbet, 1981 : 15). Cible de la dénonciation et de la satire sociale, le personnage féminin se fait l'écho des remous et des crises que traverse le Maghreb en général. L'enjeu du réalisme romanesque que nous avons posé en introduction de l'article, de la confrontation du romanesque et du social, aquiert, à ce niveau, toute sa densité qui confere à l'écriture de Serhane sa position critique face au dehors qui est nonlittéraire.

Le Soleil des obscurs foisonne d'exemples qui témoignent de la négation et de la chosification de la femme dans le système patriarcal. La femme est le signe du manque et son profil est majoritairement celui " d'une " omnisexuelle », un être dont l'attribut qui détermine toute la personnalité et les comportements est l'appareil sexuel» (Ait Sabbah, 1986 : 51). Il existe trois portraits de la femme marocaine dans le roman de Serhane : celui des complices du patriarcat, des opprimées du patriarcat et celui des véritables révoltées contre le patriarcat. La différence entre ces femmes réside dans leur façon de gérer l'oppression. Lalla Batoul et Lalla Aïcha, mères des jeunes mariés, participent au jeu de la domination patriarcale. Ayant elles-mêmes subi l'oppression à leur jeune âge et enduré les péripéties du mariage précoce (SO 119), elles manient les règles du mariage qu'elles ont arrangé en obligeant leurs enfants à s'y soumettre.

4 Titre d'un livre d'Antonio Gómez-Moriana, La subversion du discours rituel. Montréal : Le Préambule, 1985. 
Ironiquement, l'exercice systématique de l'oppression par les matriarches leur permet de "circuler" (95-96) librement dans l'espace masculin, en dehors des espaces réservés aux femmes, le bain maure et la maison, telles les guerrières algériennes de L'amour, la fantasia ou les conteuses historiennes de Loin de Médine. "L'importance et la nécessité de cette alliance condamnaient les deux femmes à être diplomates pour que ce mariage ait lieu " (SO 126). Même si le prix de cette manigance « diplomatique » est le « massacre des générations nouvelles » (175), dont celle de leurs propres enfants.

Les opprimées du patriarcat sont les jeunes filles cloîtrées, promises au mariage ou en attente d'être mariée. C'est le cas des sours de Soltane qui " grandirent dans la négation de leur corps et de leur identité. [...] Condamnées à attendre leur destin et à espérer une mort douce, leur vie passait comme une ombre discrète. [...] Comme si l'avenir avait déserté la ligne du temps pour les enfermer dans le néant " $(26 ; 101)$. C'est aussi le cas de Mina, "la fleur de champs » (69) gardée pour sa virginité (122) et perdue dans les mystères de son corps, de ses menstrues et de ses pulsions, dont la signification ne lui sera jamais donnée.

Le drame du corps féminin atteint son paroxysme lors de la cérémonie du mariage, pivot du " tabou de la virginité » (Chebel, $1995: 82 ; 100$ ) observé chez certaines tribus anciennes du Maghreb dont celle du roman de Serhane. Condition préalabe à toute union maritale et à "la transaction " (SO 143-44) de la dot versée par le mari, la virginité n'est paradoxalement pas l'apanage de la mariée. C'est tout le groupe qui en discute et s'en soucie. Elle est carnavalisée en tant que valeur ou idée spirituelle, ouvertement abordée sur la place publique du village (147) tout comme sa preuve matérielle, le sang virginal, est exigée par la foule des invités, "ivres de musiques et de fantasmes inassouvis » (149). À la limite du rire et de la tragédie, le chapitre onze décrit le mariage traditionnel dans les termes d'une " comédie grotesque " (169) et d'un "cirque " (164) de foule païenne où les " paroles sacrées f [ont] place à un répertroire riche en invectives, blasphèmes, menaces et obscénités » (178). Dans la muflerie rabelaisienne des discours tenus sur la virginité de la femme, Serhane montre le caractère obsolète et la « logique ségrégative » de la " mythologie du sang " (Chebel, 1995 : 97) dans les mours nuptiales du Maroc traditionnel. Mina, angoissée, songe à l'expérience douleureuse d'une amie (SO 151-43). Quant à Soltane, incapable d'aimer sa femme sous le vacarme de la foule qui attend que la mariée " saigne pour faire honneur aux siens en leur présentant le sang tonique de son hymène " (159), l'invite plutôt à jouer à la noce (155). Soltane transgresse, pour ainsi dire, « l'esprit 
de sérail " du groupe. Et il n'est pas jusqu’à la scène de la " défloration chamanique " (Chebel, 1995 : 83) de Mina pour montrer le pourrissement des pratiques traditionnelles et l'éternelle grisaille de la morale ancestrale communément admise. Pour calmer l'atmosphère de la haine et de désespoir (SO 166) et pour empêcher la tournure catastrophique de la cérémonie, le père de Soltane fait appel à Bouazza, le défloreur de la région (170), "l'homme à la baraka " (173) qui accomplit l'acte sexuel (171) et délivre la foule exaspérée. "La cruauté communautaire " (174) n’épargne pas Mina "violée sous [le] regard [de son mari] [...] [,] gis[ant] dans son lit comme une chose appartenant au groupe qui mange la viande de ses enfants et inscrit sur leur front des frontières qui font d'eux des cailloux ou des corps d'épargne pour les mauvais jours » (174-75).

Contrairement à tous ces portraits, c'est le personnage de Aouïcha qui incarne la révolte contre le patriarcat. Aouïcha déjoue l'oppression à travers la séduction fatale des hommes (77). Agent de désordre et de subversion à l'intérieur des labyrinthes institutionnels, elle assume sa marginalité à travers le dédoublement car elle est à la fois une prostituée et une inspiratrice de l'amour (77), une femme réelle et un personnage fantasmatique (Aïcha Kandisha). Telle Messaouda (dans Messaouda de Serhane) et Harrouda (dans Harrouda de Ben Jelloun), Aouïcha est « le symbole ambivalent du corps interdit que génèrent les systèmes répressifs. Corps interdit mais corps ludique où deviennent possibles toutes les transgressions. Il active et concentre tous les fantasmes et les désamorce en même temps dans les zones marginalisées de l'a-socialité » (Bonn \& Baumstimler, 1991 : 84).

Aouïcha, réincarnée en Aïcha Kandisha, utilise le kayd (ou la ruse) et la fitna (ou la séduction) pour contrer « la tyrannie impérieuse de la grande loi masculine » et se venger de la communauté qui l'empêchait de "suivre son destin " (SO 77). Sa vengeance prend d'autres tournures lorsqu'elle rencontre Mina au bain maure et se propose d'être sa confidente et de l'aider à guérir de ses menstrues sataniques. Sous ce pretexte, Aouïcha se venge de Si Labri, beau-père de Mina, qui l'a abandonnée dans sa jeunesse. Tout comme sa voix habite Soltane, prend régulièrement possession de son corps et se mélange parfois à la voix de sa mère (31) pour montrer "l'exercise sauvage d'une féminité [castratrice] très marquée sexuellement et maternellement » (Lacoste-Dujardin, 1985 : 170). Aouïcha abuse de la naïveté de Mina en la réduisant à un "vagin-vampire " (Ait Sabbah 116) où se transgressent tous les paramètes de la sexualité humaine. Les chapitres quatorze et quinze décrivent les séances orgiaques d'exorcisme que Aouïcha organise pour Mina en présence de fqihs et d'autres bacchans 
de son clan. Ces chapitres soulignent, en structure profonde, la plaie béante de l'ignorance. Les séances que Aouïcha désigne par " l'écriture sacrée " ou " acte de chair " (SO 215) des fqhis montrent le principe corporel dans son fonctionnement le plus abject et le plus tragique.

En pervertissant Mina, Aouïcha fait d'elle une jouisseuse condamnée par le patriarcat. D'ailleurs, Soltane, insatisfait des multiples naissances féminines qui ont précédé la venue de son garçon, accuse sa femme d'être une jouisseuse. Il lui explique que les " femmes chastes donnent naissance à des garçons [alors que [les] femmes jouisseuses ont des filles " (238). Dans l'économie de cette jouissance qui s'accomplit à travers la zina, la sexualité illicite, et à travers le péché de l'adultère, le roman distingue entre deux projets : le "projet érotique [qui] projette l'homme dans le désir féminin dont il devient l'instrument, contrairement au projet patriarcal qui est par principe axé sur le désir masculin " (Ait Sabbah 197). La sexualité dans le premier projet n'a de finalité que la satisfaction du desir, alors que dans le deuxième projet, elle a une finalité sociale et procréatrice.

Il va sans dire que le projet érotique reste très problématique dans le roman car plusieurs éléments, qui lui sont totalement opposés, le traversent, notamment la perversité, l'abject et le grotesque. En plus, si l'érotisme est souvent lié au sacré, c'est-à-dire, dans le contexte du roman, à la "face amoureuse de l'islam " (Ait Sabbah 10), tel n'est pas le cas dans Le soleil des obscurs. L'érotisme n'est sacré que s'il est illicite ou s'il est au service du projet patriarcal. Il est clair que Serhane n'aborde pas la sexualité en islam à partir de son roman. Ou s'il l'aborde indirectement, c'est pour affirmer, comme il le fait dans L'amour circoncis, que, bien que " l'Islam ait, à propos de la sexualité, quelques vues optimistes et progressistes, la société a toujours tiré un avantage de cette fonction biologique, rendant l'individu docile à son acceptation grâce à des préceptes moraux très rigides " (23). En revanche, la seule critique de l'islam apparaît, à notre avis, au niveau de l'équivalence femme-terre ou femme-objet (SO 30-31; 184). Cette équivalence trouve sa source dans un verset coranique dont la mise en texte dans le roman est diffuse ou implicite : "vos femmes sont votre semaille. Allez à votre semaille de la façon que vous voulez. Tirez-en une avance pour vous-même, en vous prémunissant envers Dieu; sachez que vous le rencontrerez : de cela porte la bonne nouvelle aux croyants » (Sourate II : verset 223). Ce verset continue à soulever plusieurs divergences sur le plan herméneutique. La féministe Ait Sabbah y voit le " caractère unilatéral de l'acte sexuel [en islam]. [...] En tant qu'entité, [la femme] est terre, elle est immobilier, elle est inerte. [...] La notion de propriété, de possession, c'està-dire de jouissance souveraine, n'est concevable que si l'élément possédé est 
privé de volonté, privé de sa capacité de contre-pouvoir » (91-92). Cependant Michel-Mansour soutient qu'il «s'agit ici d'un jeu de sens pour ce qui est du terme 'cultiver'. Ce terme a un sens littéral, celui qui désigne l'action physique de travailler la terre, et un sens figuratif ou celui qui précise [...] une cultivation de facultés intellectuelles. L'ironie se trouve là où les hommes veulent bien cultiver la femme littéralement, tandis qu'ils lui nient toute autre cultivation intellectuelle, spirituelle ou autre " (1994: 107). Si Serhane vise à subvertir l'idéologie de la femme-propriété à travers un jeu de métaphores, il nous semble que son roman renforce paradoxalement cette idéologie ou à la rigueur ne propose aucun programme d'éducation de la femme en tant que sujet pensant. La communauté des idéologues du roman est faite d'hommes (les adolescents) et l'attribut principal qui définit la femme demeure majoritairement son corps et non pas son esprit.

On tombe d'accord avec Lailla Ibnlfassi lorsqu'elle écrit que les écrivains masculins marocains, dans leur appropriation de la voix féminine, " pertétuent le même système langagier et patriarcal par le biais duquel les femmes n'atteignent jamais leur subjectivité. [...] Les femmes sont donc utilisées en tant que métaphores. [...] Par conséquent, on finit par avoir une meilleure connaissance de l'accomplissement de la subjectivité de l'auteur que celle de la femme-métaphore " (1996: 55). Si Serhane "se présente [pour plusieurs] comme annonciateur et dénonciateur du tragique féminin au Maroc » (Redouane, 1996 : 235), il n’en demeure pas moins que la femme, dans Le soleil des obscurs, n'atteint aucun héroïsme dans sa tragédie d'être. On ne saurait comparer, à titre d'exemple, l'adultère (inconscient ou involontaire) de Mina avec l'adultère de Phèdre ${ }^{5}$ de Racine ou encore avec l'adultère de " la femme adultère ${ }^{6}$ " de Camus. Il manquerait à la comparaison toute la grandeur de Phèdre et toute la féminité transcendantale du personnage camusien. De même, si la marginalité de Aouïcha indique «la difficulté d'être dans un tissu social dominé par la violence et par l'archaïsme » (Gontard 151), sa marginalité ne pourrait pas être l'étendard de la révolte ou du renouveau. Car, d'une part, Aouïcha est un personnage ambivalent et, d'autre part, «l'hyperbole du sexe » (153)

5 Voir les articles de Constant Venesoen, « Réflexions sur le péché d'adultère dans le théâtre de Racine ", Dalhousie French Studies 49 (1999) : 53-62 et de Antoine Soare, "Phèdre et les métaphores du labyrinthe : les tracés et les formes ", Dalhousie French Studies 49 (1999) : 145-57.

6 Voir l'article d'Edouard Morot-Sir, "La double transcendance du féminin et du masculin dans " La femme adultère " d'Albert Camus. " Dalhousie French Studies 19 (1990) : 51-60. 
qui domine sa marginalité réduit tout rapport interpersonnel au niveau du bas corporel.

Dans l'univers romanesque de Serhane, la représentation de la femme rejoint les visions de Michelet sur la femme, à la fois Sorcière et être fragile "s'accordant à la Nature par le rythme sanguin " (Barthes, 1964 : 121). La femme dans l'inconscient de la théologie populaire est l'être du manque et le «mal nécessaire » (SO 32). Le corps dans le labyrinthe de cette théologie, qui n'est pas purifiée des sédiments de la jahilia (période pré-islamique), est un corps problématique, "phagocité " dans ses rites, traduit dans ses actes les plus inconscients ou dans ses schèmes absolus " (Chebel, 1984 : 9). Le résultat, conclut Chebel, " tient en une phrase : l'anthropologie sexuelle au Maghreb ne serait qu'une simple phraséologie, si elle ne fixait au préalable les conditions d'émergence du statut réel du corps " (1995: 206). Ces conditions sont encore inexistantes.

\subsection{Au-delà des labyrinthes : la folie ou « la chose culturelle »}

Dans Le soleil des obscurs, la folie acquiert le statut d'une pratique de résistance aux " choses " répressives de la culture traditionnelle. La folie est littérarisée en tant que fait de société plutôt que dynamique textuelle. Elle est une réplique conséquente à l'obscurantisme social et au malaise général des individus. En effet, il est possible de réfléchir sur la folie à partir du motif du labyrinthe mis en ouvre dans le roman. Si Forest pose l'errance comme règle du labyrinthe et la transgression sexuelle comme origine du labyrinthe, dans Le soleil des obscurs, la folie s'avère le synonyme de l'errance et le dénouement cathartique de multiples transgressions sexuelles. Tous les personnages principaux sont atteints de folie. Une nef de fous "sur un océan en furie " (16) rassemble tous les adolescents qui sont, à différents degrés, hantés par « les filets de voix » (51-52) de Aouïcha la surnaturelle et par des « idées sombres mais imprécises [qui] circul[ent] dans [leurs] têtes, se bouscul[ent] parfois, se pouss[ent] comme des vers, [les] conduisant vers la folie ou la démence » (51). Plus que ses amis, Soltane est "guetté par la folie comme son grand-père " (20) et son père, amoureux fou de Aouïcha. Soltane est "fou " et " possédé " (108) par Aouïcha qui prend la forme de silhouettes étranges (130) pour lui faire son apparition. La folie de Soltane est de nature sexuelle : ses pulsions sont décrites comme une "espèce de démangeaison, comme la lèpre ou la folie " (98). La défloration dramatique de sa femme lors de sa nuit de noces le précipite dans un " tunnel [de folie] qui était sans limites" (177). Mina, 
qui se pense " atteinte de folie " (205) à cause de ses menstrues, s'adonne aux écritures sacrées des fqihs sous l'égide de Aouïcha et finit par donner plusieurs naissances pendant l'absence de Soltane parti dans la ville où il travaille, en tant que chauffeur de taxi, dans "la vente du sexe " (235). Le jour du baptême de son fils, il rentre à la maison avec un enfant bâtard délaissé par sa mère dans son taxi. Le désordre et la confusion entre familles, à cause de cet enfant suspecté d'être le fils illégitime de Soltane, finit en cour et après plusieurs analyses médicales et tests de paternité, Soltane est diagnostiqué stérile. Il "sombre dans [une] folie " (254) irrévocable sans comprendre le mystère de ces multiples enfants dont il est le père. À "sa sortie de l'hôpital ", il devient un conteur sur la place publique de son village et il continue à être poursuivi par la "silhouette fragmentée " (253) de Aouïcha; seuls ses "yeux gardaient une expression de révolte triste où s'étaient accumulés tous les signes d'une misère sans limites et les indices d'une douleur inhumaine " (254).

Dans l'emboîtement de ces diverses folies, il est intéressant de souligner la révolte des belles-mères contre leurs maris en conclusion du roman. La folie de Soltane et le scandale de l'enfant trouvé indignent les belles-mères qui se disputent et demandent d'être répudiées (251) pour être libres. La violence et le délire des belles-mères (252) sont une sorte de folie libératrice visant à restituer la réalité individuelle de chacune. Ainsi le roman de Serhane est travaillé par " le double discours [de la folie] qui permet à ceux qui l'exercent la survie psychologique et la possibilité de se révolter subrepticement " (Mésavage, 1998 : 53). D’un côté, il y a la folie imposée par une société hypocrite qui projette dans l'indivis et dans la déchéance identitaire et, d'un autre, il y a la " bouffée délirante » des marginaux de la société, " idiots du village ou délirants mystiques [...] [qui] disent la vérité du groupe car ils n'y sont pas assujettis; ils sont dedans et dehors à la fois » (Ifrah, 1980 : 19). Soltane est une sorte d'« El Mejdoub [personnage typique des contes populaires marocains] qui, au péril de sa vie, jour et nuit crie la misère du peuple et sa soif de justice; sa folie est [donc] sagesse. » (19). La "societé schizomorphe " (Mésavage 62) du roman de Serhane reflète le malaise des mutations et des profondes remises en cause de tout un système disloqué ou en état de dislocation. C'est dans ce sens, par exemple, que la question des racines et des origines revient dans le roman en rapport avec la question de l'urbanisme et de l'exode rural. (SO 209). Chikhi catalogue sous le "graphe $\mathrm{O}$ » toutes les « histoires d'O. rigine » et d'identité dans le roman maghrebin contemporain. Dans Le soleil des obscurs, c'est « l'O de l'Obscur » qui résume « la blessure innommée [qui] 
se réveille parle parle comme jeune vie dissimulée par les ombres » (Chikhi, 1996 : 42) des personnages effacés.

En bouleversant la donne de l'écriture réaliste à travers la parole limite de la folie, du fantastique et même du grotesque, Serhane soulève les insuffisances du grégarisme et du discours collectif qui placent l'individu dans une impasse existentielle et tracent la victimologie de son destin. Le labyrinthe physique et psychologique est-il surmonté à la fin du roman? Il nous semble que le labyrinthe se referme sur les adolescents du village qui dansent de joie (SO 255) dans l'illusion d'un horizon chargé de pluie et de délivrance : " $[\mathrm{n}]$ ous étions comme ivres sous le poids de ces nuages qui ne voulaient pas crever entre nos bras nus. La pluie n'arriva pas. Un vent infidèle nettoya notre ciel qui retrouva sa sérénite aussi brusquement qu'il l'avait perdue, et notre espoir tomba en décrépitude » (255-56). C'est dans ces termes sinistres que s'achève le roman, avec le sentiment que l'épreuve de l'altérité ou de la subversion qui s'effectue au sein de chaque labyrinthe retrouve le chemin de l'origine, l'impasse du point de départ. Les adolescents du roman portent fatalement et incurablement " le soleil noir de la mélancolie " caractéristique de l'El desdichado nérvalien. Le sens de l'épreuve les aura conduits à la rencontre d'un destin bouclé que leur vie démaillée aura ironiquement tracé.

Et c'est peut-être dans le partage de la folie et de l'illusion que Serhane trace l'itinéraire du changement social qui serait impensable sans la traversée de l'écriture romanesque vers une certaine utopie, en deçà du discours orthodoxe qui interprète toute innovation par la création (bida'a) dans les termes d'un errement (dalala).

\section{Bibliographie}

Ait Sabbah, Fatna. La femme dans l'inconscient musulman. Paris : Albin Michel, 1986.

Bakhtine, Mikhaïl. L'ouvre de François Rabelais et la culture populaire au Moyen Âge et sous la Renaissance. Paris : Gallimard, 1970.

-. Esthétique de la création verbale. Paris : Gallimard, 1984.

Barthes, Roland. Essais critiques. Paris : Seuil, 1964.

Ben Jelloun, Tahar. Harrouda. Paris : Denoël, 1973.

-. L'enfant de sable. Paris : Seuil, 1985.

-. La nuit sacrée. Paris : Seuil, 1987.

Bonn, Charles. «Schémas psychanalytiques et roman maghrébin de langue française. "Psychanalyse et texte littéraire au Maghreb. Eds. Charles Bonn and Yves Baumstimler. Paris : L’Harmattan, 1991. 11-23. 
Bonn, Charles and Yves Baumstimler. Psychanalyse et texte littéraire au Maghreb. Paris : L'Harmattan, 1991.

Camus, Albert. La chute. Paris : Gallimard, 1998.

-. La peste. Paris : Gallimard, 2000.

Chebel, Malek. Le corps dans la tradition au Maghreb. Paris : PUF, 1984.

--. L'esprit de sérail. Mythes et pratiques sexuels au Maghreb. Paris : Payot, 1995.

Chikhi, Beïda. Maghreb en textes. Écritures, histoire, savoirs et symboliques. Essai sur l'épreuve de modernité dans la littérature de langue française. Paris : L'Harmattan, 1996.

Déjeux, Jean. Le sentiment religieux dans la littérature maghrébine de langue française. Paris : L'Harmattan, 1986.

Delcambre, Anne-Marie. Liislam. Paris : La Découverte, 1999.

Des Rosiers, Joël. " Présentation. Médecine et littérature : cliniciens ès lettres. » Spirale mai-juin. $2000: 12$.

Djebar, Assia. L'amour, la fantasia. Casablanca : EDDIF, 1992.

—. Loin de Médine. Filles d'Ismaël. Paris : Albin Michel, 1991.

Ferchiou, Sophie. «Introduction : l'islam pluriel dans le Maghreb contemporain. " Lislam pluriel au Maghreb. Ed. Sophie Ferchiou. Paris : CNRS Éditions, 1996. 1-10.

Forest, Philippe. Textes et labyrinthes. Mont-de-Marsan : Éditions InterUniversitaires, 1995.

Galland, Antoine. Trans. Les Milles et une Nuits. 3 vols. Paris : GarnierFlammarion, 1965.

Gómez-Moriana, Antonio. La subversion du discours rituel. Montréal : Le Préambule, 1985.

Gontard, Marc. Le moi étrange : littérature marocaine de langue française. Paris : L'Harmattan, 1993.

Ibnlfassi, Laïla. "L'appropriation de la voix féminine par les écrivains masculins marocains. "La traversée du français dans les signes littéraires marocains. Actes du colloque international de l'université York, Toronto 20-23 avril, 1994. Eds. Yvette Bénayoun-Szmidt, Hédi Bouraoui and Najib Redouane. Toronto : La Source, 1996. 55-63.

Ifrah, Albert. Le Maghreb déchiré. Claix : La pensée sauvage, 1980.

Khatibi, Abdelkebir. Amour bilingue. Saint-Clément-de-Rivière : Fata Morgana, 1983.

Kourouma, Ahmadou. Les soleils des indépendances. Paris : Seuil, 1970.

Lacoste-Dujardin, Camille. Des mères contre les femmes. Maternité et patriarcat au Maghreb. Paris : La Découverte, 1985. 
Médecine et littérature. Cliniciens ès lettres. Spec. issue of Spirale mai-juin. $2000: 12-25$.

Memmi, Albert. Portrait du colonisé suivi de Les Canadiens français sont-ils des colonisés? Montréal : l'Étincelle, 1972.

Mésavage, Matilde. "Le "double discours" dans Le soleil des obscurs d'Abdelhak Serhane. "Le Maghreb littéraire. Revue canadienne des littératures maghrébines 2.3 (1998) : 51-63.

Michel-Mansour, Thérèse. La portée esthétique du signe dans le texte maghrébin. Paris : Publisud, 1994.

Nerval, Gérard. "El desdichado. » Poésies. Ed. Albert Béguin. Lausanne : Mermod, 1947. 27.

Nisbet, A.-M. " Le personnage féminin dans le roman maghrébin de langue française. "Écriture française 3. 6-7 (1981): 14-17.

Radi, Saâdia. "Croyance et référence : l'utilisation de l'islam par le Fqih et par la suwãfa à Khénifra (Maroc). "L'islam pluriel au Maghreb. Ed. Sophie Ferchiou. Paris : CNRS Éditions, 1996. 189-99.

Redouane, Najib. «Abdelhak Serhane : annonciateur et dénonciateur du tragique féminin au Maroc. "La traversée du français dans les signes littéraires marocains. Actes du colloque international de l'université York, Toronto 20-23 avril, 1994. Eds. Yvette Bénayoun-Szmidt, Hédi Bouraoui and Najib Redouane. Toronto : La Source, 1996. 219-36.

Riffaterre, Michael. "L'illusion référentielle. " Littérature et réalité. Eds. Gérard Genette and Tzvetan Todorov. Paris : Seuil, 1982. 81-90.

Sartre, Jean-Paul. La nausée. Paris : Gallimard, 1997.

Serhane, Abdelhak. Messaouda. Paris : Seuil, 1983.

-. Le soleil des obscurs. Paris : Seuil, 1992.

-. Les prolétaires de la haine. Paris : Publisud, 1995.

-. L'amour circoncis. Casablanca : EDDIF, 1995.

-. Le deuil des chiens. Paris : Seuil, 1998.

Sjursen, Nina. "Voix dans La peste ou la rencontre polyphonique avec le mal. "Point de rencontre: le roman. Actes du colloque international d'Oslo, 7-10 septembre, 1994. Vol. 2. Ed. Juliette Frølich. Oslo : Conseil norvégien de la recherche scientifique, 1995. 321-31.

Soare, Antoine. « Phèdre et les métaphores du labyrinthe : les tracés et les formes. " Dalhousie French Studies 49 (1999) : 145-57.

Venesoen, Constant. «Réflexions sur le péché d'adultère dans le théâtre de Racine." Dalhousie French Studies 49 (1999) : 53-62. 